\title{
Statistical Analysis of Periodic Oscillations in LASCO Coronal Mass Ejection Speeds
}

\author{
G. Michalek ${ }^{1}$ - A. Shanmugaraju ${ }^{2}$ N. Gopalswamy ${ }^{3}$. \\ S. Yashiro ${ }^{3} \cdot$ S. Akiyama ${ }^{3}$
}

Received: 18 May 2016 / Accepted: 30 September 2016 / Published online: 26 October 2016

(C) The Author(s) 2016. This article is published with open access at Springerlink.com

\begin{abstract}
A large set of coronal mass ejections (CMEs, 3463) has been selected to study their periodic oscillations in speed in the Solar and Heliospheric Observatory (SOHO) mission's Large Angle and Spectrometric Coronagraph (LASCO) field of view. These events, reported in the SOHO/LASCO catalog in the period of time 1996-2004, were selected based on having at least 11 height-time measurements. This selection criterion allows us to construct at least ten-point speed-distance profiles and evaluate kinematic properties of CMEs with a reasonable accuracy. To identify quasi-periodic oscillations in the speed of the CMEs a sinusoidal function was fitted to speed-distance profiles and the speed-time profiles. Of the considered events $22 \%$ revealed periodic velocity fluctuations. These speed oscillations have on average amplitude equal to $87 \mathrm{~km} \mathrm{~s}^{-1}$ and period $7.8 R_{\odot} / 241 \mathrm{~min}$ (in distance/time). The study shows that speed oscillations are a common phenomenon associated with CME propagation implying that all the CMEs have a similar magnetic flux-rope structure. The nature of oscillations can be explained in terms of magnetohydrodynamic (MHD) waves excited during the eruption process. More accurate detection of these modes could, in the future, enable us to characterize magnetic structures in space (space seismology).
\end{abstract}

Keywords Sun: solar activity $\cdot$ Sun: coronal mass ejections

\section{Introduction}

Coronal mass ejections (CMEs) are large expulsion of magnetized plasma from the Sun. They are actively studied because of their significant effect on the Earth's space. CME dynamics can be explained by magnetohydrodynamic models based on flux rope structure (e.g. Chen et al., 1997, 2000; Wood et al., 1999; Krall et al., 2001; Chen and Krall,

\footnotetext{
$凶$ G. Michalek

michalek@oa.uj.edu.pl

1 Astronomical Observatory of Jagiellonian University, Cracow, Poland

2 Department of Physics, Arul Anandar College, Karumathur 625 514, India

3 NASA Goddard Space Flight Center, Greenbelt, MD, USA
} 
2003; Krall, 2007). These models allow the inclusion of magnetic forces that drive the motion of the flux rope relative to the solar wind (see Forbes, 2000 and references therein). Furthermore, the propagation of CMEs in the solar corona and interplanetary medium is also strongly affected by the aerodynamic drag due to their interaction with the ambient magneto-plasma (Cargill et al., 1996; Lindsay et al., 1999; Gopalswamy et al., 2000, 2001; Cargill, 2004). The interplay between these forces is not fully understood (e.g. Vršnak, 2006; Vršnak et al., 2004). CMEs that are faster than the solar wind tend to decelerate, whereas events slower than the solar wind accelerate (Gopalswamy et al., 2000; Yashiro et al., 2004; Vršnak et al., 2004). During the solar maximum, the situation may be more complicated and the dynamics of CMEs is additionally affected by interaction between successive events (Gopalswamy et al., 2002; Wang, Wu, and Gopalswamy, 2005). As a result of these collisions CMEs are abruptly accelerated or decelerated.

Krall et al. (2001) reported quasi-periodic oscillations in the speed of several CMEs. These oscillations were pronounced in C3 Large Angle and Spectrometric Coronagraph (LASCO) images and had approximate periods from 4 to 6 hours. In the flux rope models such oscillations appear if a stable flux rope structure is weakly perturbed. The presence of these oscillations proves that CMEs have an organized magnetic structure with normal modes of oscillations (Chen, 1989; Cargill, Chen, and Garren, 1994). Therefore the reconnection of the magnetic field during the ejection of a CME should excite these modes of oscillation with changing parameters as the flux rope expands. Periodic velocity and intensity oscillations are well established in solar quiescent prominences which in many cases are progenitors of CMEs (e.g. Joarder, Nakariakov, and Roberts, 1997). These oscillations have a range of periodicity from a few minutes up to the order of an hour.

Recently, Shanmugaraju et al. (2010) have carefully considered 116 CMEs with at least 10 height-time measurements in the LASCO field of view (LFV). They found 15 events exhibiting clear quasi-periodic patterns in speed-distance profiles. The amplitudes of oscillations were found in the range $157-418 \mathrm{~km} \mathrm{~s}^{-1}$ and with periods in the range $48-240 \mathrm{~min}$.

The main goal of the present study is to confirm weather speed oscillation is a common feature associated with CME propagation using a much larger sample than that used in previous studies. For this purpose we analyzed the speed-distance profiles for all the CMEs reported in the Solar and Heliospheric Observatory (SOHO) LASCO catalog (Gopalswamy et al., 2009) during 1996 to 2004. We determined regular velocity fluctuations by fitting sinusoidal functions to the speed-distance profiles. About $37 \%$ of the events considered in this study revealed reasonable speed oscillation patterns. We also performed a statistical analysis of the oscillation attributes.

This paper is organized as follows. In Section 2, the method used for this study is described. Results of our analysis are presented in Section 3. Finally, the discussion and conclusions are included is Section 4.

\section{Method to Analyze Oscillation in the Speed of CMEs}

In this section we describe briefly the method used to determine oscillations in the speed of CMEs.

\subsection{Data Selection}

We consider a large sample of 9496 CMEs detected by the LASCO coronagraphs from January 1996 to December 2004 and reported in the SOHO/LASCO catalog ${ }^{1}$ (Yashiro et al.,

\footnotetext{
${ }^{1}$ http://cdaw.gsfc.nasa.gov/CME_list/.
} 
2004; Gopalswamy et al., 2009). This period covers almost one cycle of solar activity. It is obvious that a reliable analysis of oscillations requires an adequate sample of observations. Therefore, we consider only the events having at least 11 height-time measurements (at least 10 velocity-height points) reducing the sample size to 3463 events. Unfortunately, this criterion makes our sample biased to the speed range up to $1500 \mathrm{~km} \mathrm{~s}^{-1}$. The fastest CMEs (with velocity $>1500 \mathrm{~km} \mathrm{~s}^{-1}$ ) are not suitable for oscillation considerations because they have only a few height-time measurements within the LASCO field of view.

For the present study we utilized the height-time measurements and data included in the SOHO/LASCO CME catalog. The propagation of CMEs is traced manually and errors in measurements can be large and unpredictable (Wen, Maia, and Wang, 2007). These random errors significantly affect the determination of CME velocity and acceleration. In the present study, to minimize uncertainties of measurements, a large sample of CMEs having at least eleven height-time measurements was selected.

\subsection{Determination of Oscillations}

Our analysis is based on speed-distance or speed-time profiles of CMEs obtained from the height-time measurements included in the SOHO/LASCO catalog. Instantaneous speeds of CMEs were calculated using the following formula:

$$
v_{i+\frac{1}{2}}=\frac{r_{i+1}-r_{i}}{t_{i+1}-t_{i}}
$$

where $\left(r_{i}, t_{i}\right)$ represents the $i$ th height-time data point and $v_{i}$ is the CME speed at the time $t_{i}$. The velocities evaluated in this way were used in our further analysis. As in Shanmugaraju et al. (2010) we assigned minimum speed errors at $30 \mathrm{~km} \mathrm{~s}^{-1}$ and $80 \mathrm{~km} \mathrm{~s}^{-1}$ in the $\mathrm{C} 2$ and $\mathrm{C} 3$ field of view (FOV), respectively. Next a sinusoidal function, including five free parameters, was fitted to the speed-distance or speed-time profiles. The function has the form

$$
v(x)=a_{0} \sin \left(a_{1} x+a_{2}\right)+a_{3}+a_{4} x,
$$

where $a_{0}$ is the amplitude of oscillations, $a_{1}$ is the frequency of oscillations, $a_{2}$ is the phase, $a_{3}$ is the starting velocity, $a_{4}$ is the acceleration, and $x$ can express distance or time. The parameters $a_{2}$ and $a_{3}$ are not important for our study and they are not discussed further. In many cases CME velocities show a systematic increase or decrease in the LASCO FOV, therefore, in addition to the pure oscillation pattern, the last term in Equation (2) as introduced. The parameter $a_{4}$ has a different meaning depending on whether we consider speed versus distance or time. In the first case $a_{4}$ express a linear change of velocity with distance. In the second case it express acceleration. It is important to note that the acceleration expressed by the parameter $a_{4}$ differs from that reported in the SOHO/LASCO catalog. We considered also more complicated sinusoidal function having two more free parameters describing a change of amplitude and in the frequency of oscillations with distance. However, the results were not promising. In the distance range covered by the LASCO coronagraphs a simpler sinusoidal fitting (Equation (2)) works better. It is important to use an accurate fitting procedure. We have tested different approaches including Monte Carlo simulations. All of the methods worked equally well. After considerations we decided to employ the following method. For the numerical fitting procedure an IDL routine (mpfitfun.pro) and Monte Carlo simulation was utilized. The mpfitfu.pro program is a non-linear routine based on the least-square fitting method (Markwardt, 2009). This procedure is fast but sensitive to starting parameters. To obtain accurate results it is necessary to choose appropriate initial 


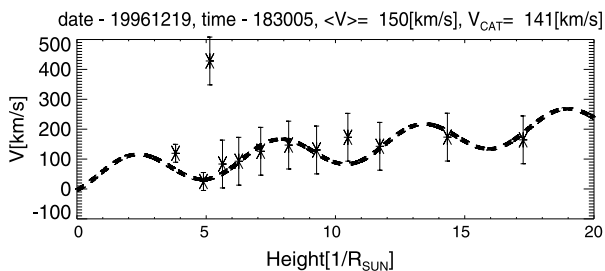

date -20000307 , time $-163005,\langle\mathrm{~V}\rangle=678[\mathrm{~km} / \mathrm{s}], \mathrm{V}_{\mathrm{CAT}}=643[\mathrm{~km} / \mathrm{s}]$

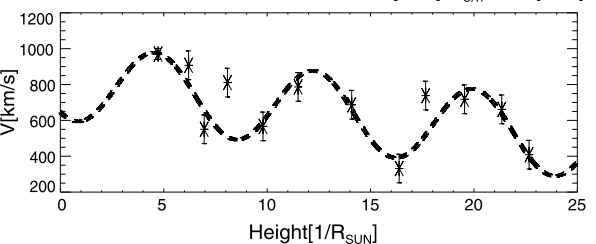

date -19961219 , time $-183005,\langle\mathrm{~V}\rangle=150[\mathrm{~km} / \mathrm{s}], \mathrm{V}_{\mathrm{CAT}}=141[\mathrm{~km} / \mathrm{s}]$

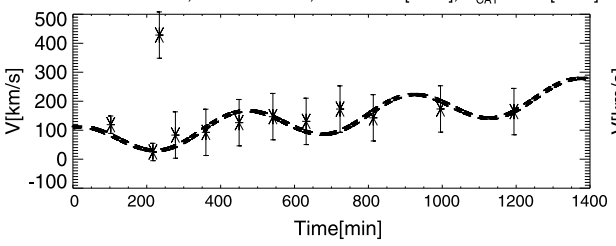

date -20000307 , time $-163005,\langle\mathrm{~V}\rangle=678[\mathrm{~km} / \mathrm{s}], \mathrm{V}_{\mathrm{CAT}}=643[\mathrm{~km} / \mathrm{s}]$

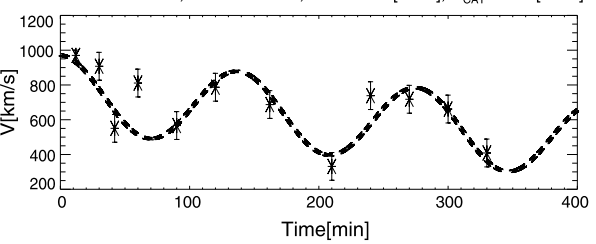

Figure 1 Examples of a fitted curves (dashed lines) to speed-distance/time profiles for two CMEs. Left panels show a CME on 09/12/1996 and right panels show a CME on 07/03/2000, respectively. Upper panels display speed-distance profiles and lower panels speed-time profiles, respectively. For these CMEs we obtained the following parameters of oscillations: for 09/12/1996 $a_{0}=53 \mathrm{~km} \mathrm{~s}^{-1}, a_{1}=5.5 R_{\odot}, a_{4}=9.2 \mathrm{~m} \mathrm{~s}^{-2}$; and for $07 / 03 / 2000 a_{0}=216 \mathrm{~km} \mathrm{~s}^{-1}, a_{1}=7.5 R_{\odot}, a_{4}=-13.2 \mathrm{~m} \mathrm{~s}^{-2}$. Error bars show the minimum speed error for $\mathrm{C} 2$ and $\mathrm{C} 3$ instruments.

conditions. For this purpose, using Monte Carlo simulations a large set of starting parameters was simulated for each CME. These parameters were next used for the IDL fitting routine. This procedure allowed us to fit the sinusoidal function with high accuracy. Without imposing any limits on the free parameters, the fitting procedure was applied to all the considered CMEs. Then, having the parameters of the sinusoidal fitting procedure we can decide if a given CME really exhibits quasi-periodic fluctuations of velocity. We assume that oscillations are reasonable when the amplitude is in the range $\left(30 \mathrm{~km} \mathrm{~s}^{-1}\right.$ to $\left.400 \mathrm{~km} \mathrm{~s}^{-1}\right)$, the space period of oscillations is lower than $16 R_{\odot}$ and the time period of oscillation is more than $60 \mathrm{~min}$. The lower limit in the amplitude is enforced by the minimal speed errors and the upper limit is to avoid the situation when the amplitude of oscillations is larger than the actual speed of an event. The limits in the period of oscillation occur because of the time cadence of LASCO coronagraphs and the space range of the LASCO coronagraphs. The full fitting procedure was only applied to the speed-time profiles. In the case of the speed-distance profiles we fitted only two parameters $\left(a_{2}\right)$ and $\left(a_{3}\right)$. We assumed that the amplitude of oscillations $\left(a_{0}\right)$ and the acceleration $\left(a_{4}\right)$ must be the same for both the profile of speed-distance and of speed-time. The space period of oscillation $\left(a_{1}\right)$ is simply equal to the time period of oscillation multiplied by the average velocity of a given event. Using our procedure we were able to select $773 \mathrm{CMEs}$ showing clear periodic speed fluctuations. These oscillations are not produced by the applied procedure. The shape of the sinusoidal function and the fitting method do not impose the fitting of an oscillating function to the velocity-distance profiles.

In Figure 1 two examples of CMEs showing clear quasi-periodic fluctuations of velocity are presented. They are typical examples. For these events, we see that oscillation patterns overlap on the systematic increase or decrease of velocity for these CME examples. In spite of the fact that not all points are very well fitted to the model curves the oscillation patterns are clear. Outlier points, e.g. the one for the CME on 09/12/1996, result from errors in the manual determination of height-time points.

Goodness of fit statistics can be determined using the differences between the data points and the fit (the residuals). For each CME we determined the average value of the residuals. 
Figure 2 Distribution of relative error. The relative error for each CME was obtained by comparison of the average residual value to the amplitude of oscillation.

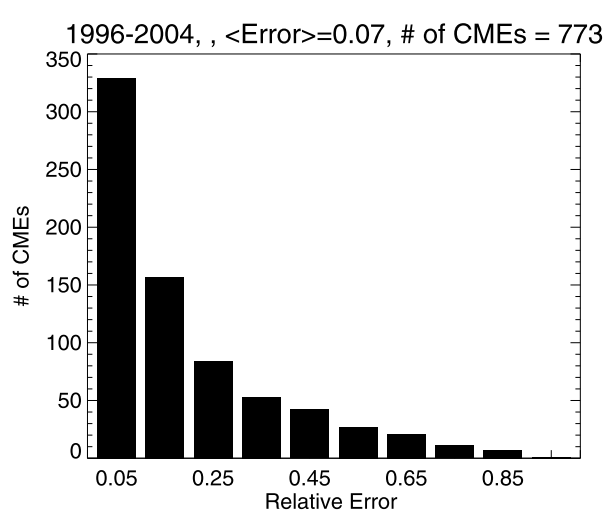

The average value of the residuals compared to the amplitudes of oscillation can be a good estimator of the goodness of fit for a given CME. Figure 2 shows the distribution of relative error obtained in this way. The vast majority of CMEs have very small fitting errors (the average value of the relative errors is 0.07 ). Only $\approx 50 \mathrm{CMEs}$ have relative errors comparable to the amplitudes of oscillations.

\section{Results}

\subsection{General Properties of Oscillations}

Distributions of the most important attributes of the oscillations are displayed in Figure 3. The successive panels show the distributions of the basic attributes (amplitude, space period, time period and acceleration). The amplitudes of oscillations can be very large (up to $391 \mathrm{~km} \mathrm{~s}^{-1}$ ). The average amplitude of oscillations is almost $100 \mathrm{~km} \mathrm{~s}^{-1}$. Due to the resolution of the LASCO images, as mentioned previously, we considered only oscillations with amplitudes larger than $30 \mathrm{~km} \mathrm{~s}^{-1}$. Periods of oscillations cover a wide range of values (from $0.94 R_{\odot}$ up to $15.97 R_{\odot}$ for space periods and from 90 up to 824 min for time periods). For the extreme values of parameters of oscillation the accuracy of the sinusoidal fitting was verified. The average value of period is consistent with the previous results $\left(7.8 R_{\odot}\right.$ and $241 \mathrm{~min}$ for the space and time period, respectively). The last panel in Figure 3 displays the acceleration evaluated at the base of the speed-distance profiles. It is different from that reported in the SOHO/LASCO catalog. It was determined from the velocities obtained using Equation (1). CMEs showing an oscillation pattern mostly move with constant velocities. The average value of the acceleration is $2.0 \mathrm{~m} \mathrm{~s}^{-2}$. However, we can observe a small excess of CMEs having a positive acceleration (the median is $1.8 \mathrm{~m} \mathrm{~s}^{-2}$ ).

\subsection{Characteristics of CMEs Showing Speed Oscillations}

Distributions of the most important attributes of CMEs revealing speed oscillations are displayed in Figure 4. The successive panels show distributions of velocities, widths, absolute latitudes and accelerations obtained from a quadratic fit to the height-time points. Instead of the central position angle (CPA) reported in the catalog we consider the absolute latitude, to have a clear comparison between equatorial and polar events. The CMEs attributes cover a wide range of values $\left(87-1352 \mathrm{~km} \mathrm{~s}^{-1}\right.$ for velocity, $3-314$ degrees for width and 
(a) $1996-2004,<$ Amplitude $>=87 \mathrm{~km} / \mathrm{s}$, median=74km $/ \mathrm{s}$, stdev=47, \# of CMEs $=773$

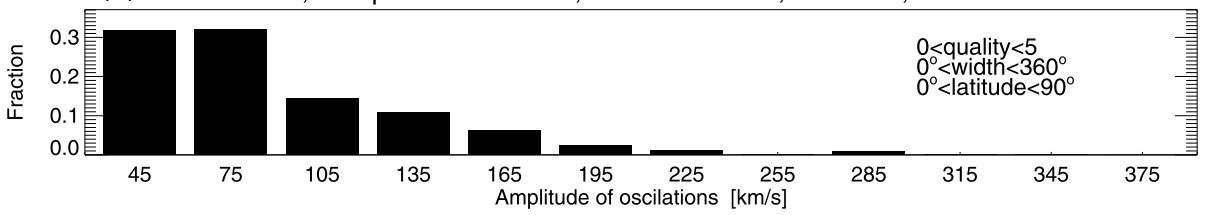

(b) $\quad 1996-2004,<$ Period $>=7.8 \mathrm{R}_{\mathrm{SUN}}$, median $=7.5 \mathrm{R}_{\mathrm{SUN}}$, stdev=3.4, \# of CMEs $=773$

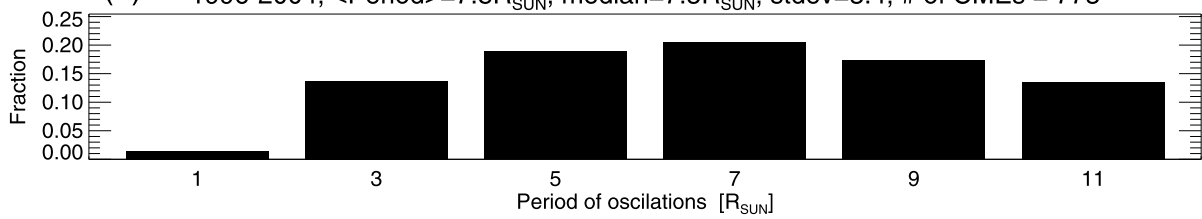

(c) $1996-2004,<$ Period $>=241 \mathrm{~min}$, median $=228 \mathrm{~min}$, stdev $=10$, \# of CMEs $=773$

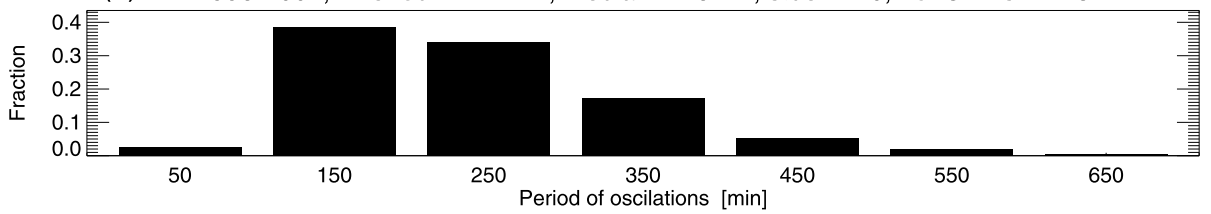

(d) $1996-2004,<$ Acceleration $>=2.0 \mathrm{~m} / \mathrm{s}^{2}$, median $=1.8 \mathrm{~m} / \mathrm{s}^{2}, \mathrm{stdev}=12.3$, \# of CMEs $=773$

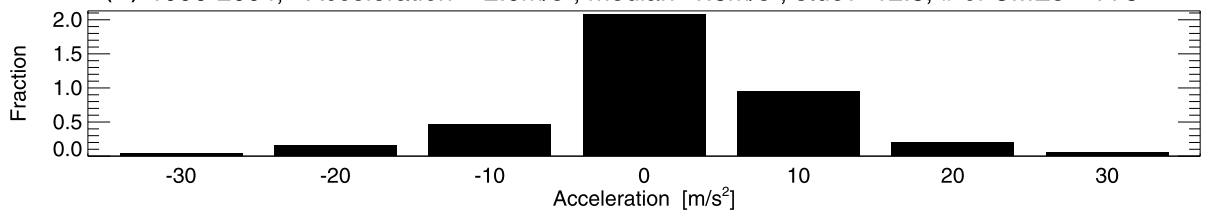

Figure 3 Distributions of the most important attributes of the speed oscillations. In the successive panels there are distributions of the amplitude of oscillation (parameter $a_{0}$ ); the space period of oscillation (parameter $a_{1}$ ); the time period of oscillation (parameter $a_{1}$ ); and the acceleration of CME (parameter $a_{4}$ ). Basic statistics (mean, median, standard deviation) are reported at the top of the figures.

$-68-90 \mathrm{~m} \mathrm{~s}^{-2}$ for acceleration) implying that speed oscillations are a common feature accompanying propagation for a variety of classes of events. This suggests that all CMEs should have a similar magnetic flux-rope structure favorable to excite normal modes of oscillations. The distributions of CME attributes resemble those obtained for the general populations of CMEs (Yashiro et al., 2004). Only the widths of the oscillating CMEs seem to be on average larger in comparison with the average width of the general population of CMEs. Probably this difference is caused by our selection criterion. Narrower events usually have a lower quality of observations and may be excluded from our study due to their not having a sufficient number of height-time points.

\subsection{Quality of CMEs}

In the $\mathrm{SOHO} / \mathrm{LASCO}$ catalog a quality index with values ranging from 0 to 5 (with a step 1) was introduced on the basis of brightness and sharpness of the leading edge of a given CME. Figure 5 displays the fractions of CMEs in our study with a respective quality index. In the upper panel we present the fractions for CMEs having a clear oscillation pattern (773 events). $70 \%$ of CMEs with an oscillation pattern have a quality index $\geq 3$. The bottom panel presents the fraction of CMEs with oscillations in speed having a given quality index 
(a) 1996-2004, <Velocity $>=399 \mathrm{~km} / \mathrm{s}$, \# of CMEs $=773$

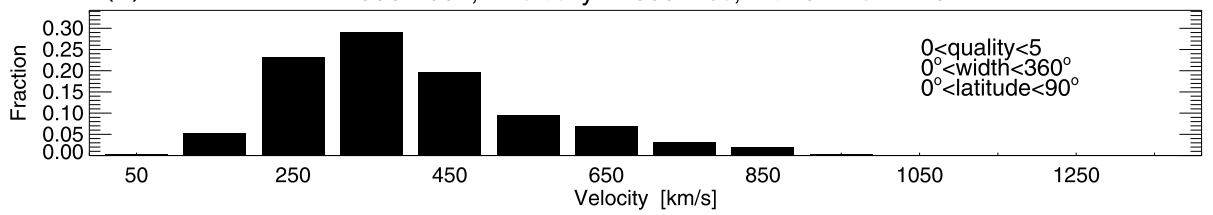

(b)

1996-2004, <Width>=79Deg, \# of CMEs = 773

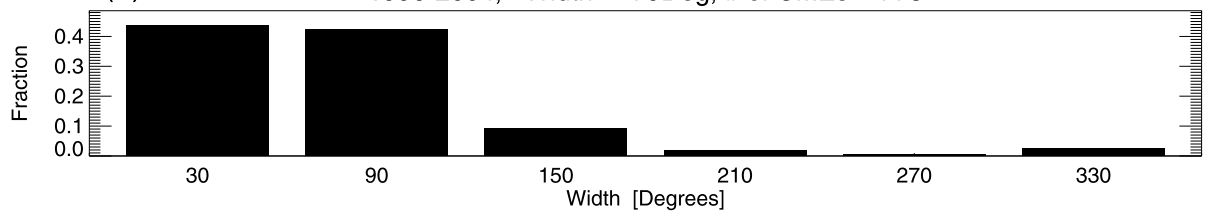

(c) 1996-2004, <|Latitudel>= 33Deg, \# of CMEs = 773

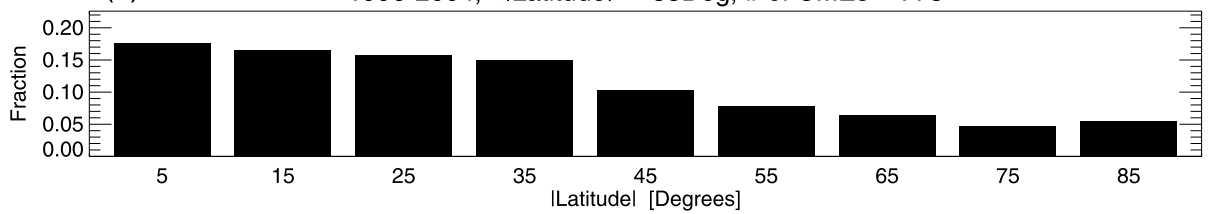

(d) 1996-2004, $<$ Acceleration $>=2 \mathrm{~m} / \mathrm{s}^{2}$, \# of CMEs $=773$

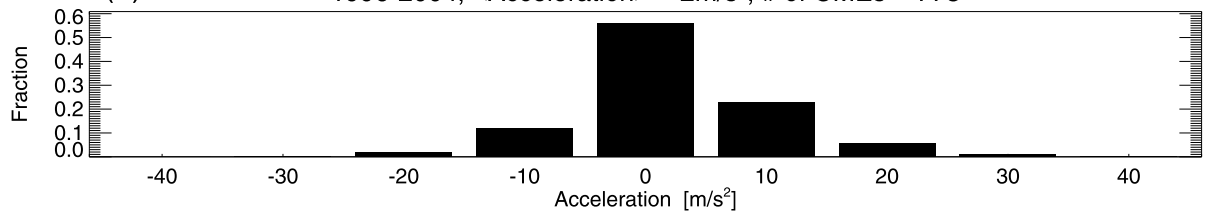

Figure 4 Distributions of the most important attributes of CMEs showing speed oscillations. In the successive panels there are distributions of: velocities, widths, absolute latitudes, and accelerations of CMEs.

with respect to all CMEs in this study having the same quality index. Figure 5 demonstrates that larger quality index CMEs are favored for oscillation detection. More than $20 \%$ of all the considered CMEs with quality index $\geq 3$ revealed clear oscillations in speed. On the other hand, only $15 \%$ of all the considered CMEs having an index equal to zero demonstrated oscillations. This fraction is comparable with that for the higher quality events but we have to remember that poor events were mostly eliminated at the initial stage of our considerations (by the assumption that CMEs need to have at least 11 height-time points). The detection of oscillations depends strongly on the quality of observations. This dependence confirms that the recorded oscillations are a real physical phenomenon. If the oscillations were random phenomenon, this correlation should not be observed.

\subsection{Oscillation Appearance Frequency}

Figure 6 shows the oscillation appearance frequency during the considered period of time. Oscillations were recorded mostly frequently during the maximum of solar activity (2001). This is evident from the absolute and fractional diagrams. During maximum about $15 \%$ of all the CMEs revealed speed oscillations. This suggests that more powerful events are more likely to excite speed fluctuations. On average more than $5 \%$ of CMEs reported in the $\mathrm{SOHO/LASCO}$ catalog revealed a speed oscillation pattern. 
Figure 5 Fractions of CMEs with a given quality index. The upper panel displays the fraction of CMEs with a given quality index with respect to all the events showing an oscillation pattern (1283). The bottom panel displays the fraction of CMEs with oscillations in speed having a given quality index with respect to all the considered events with the same quality index. (a) 1996-2004, \# of CMEs $=773$

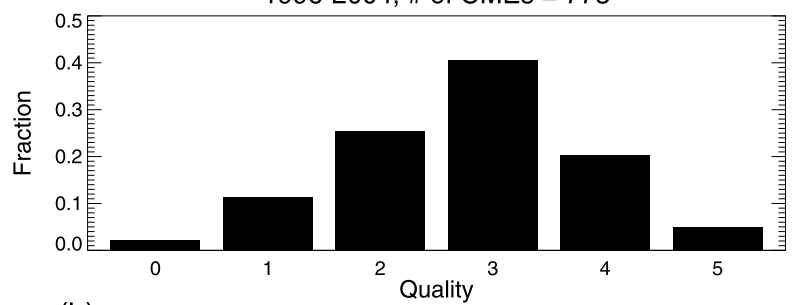

(b) 1996-2004, \# of CMEs $=3463$

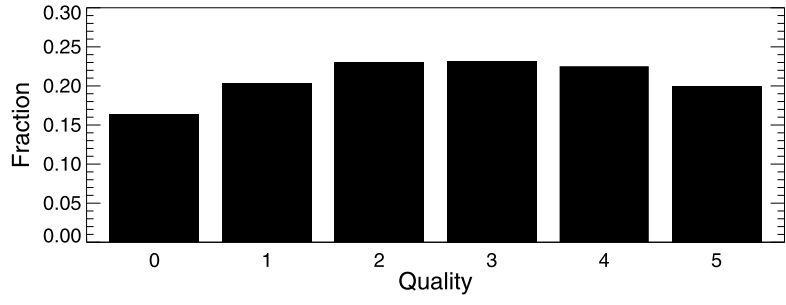

(a) $1996-2004, \#$ of CMEs $=773$ of CMEs with oscillations in speed. The upper panel displays the absolute and the bottom panel displays the fractional oscillation appearance frequency, respectively.

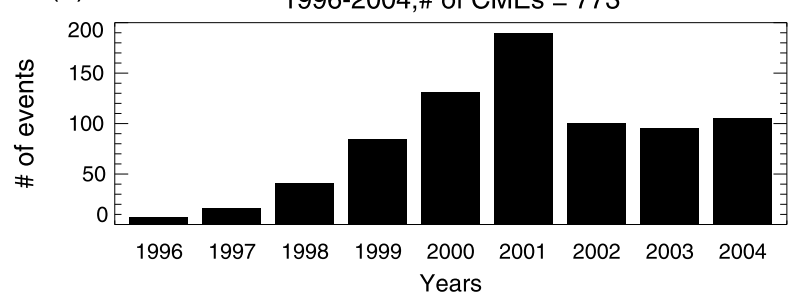

(b) 1996-2004,\# of CMEs = 773

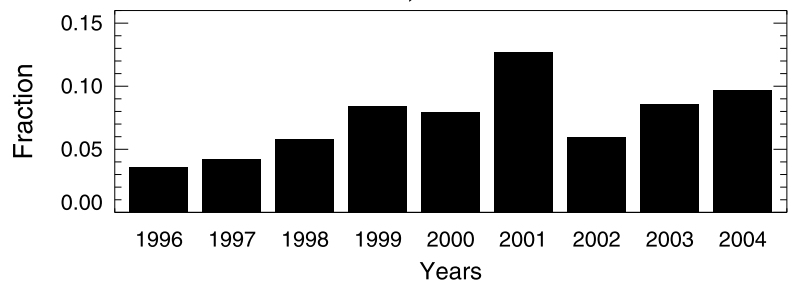

\subsection{Oscillation Attributes Versus CME Attributes}

Figure 7 shows scatter plots of the amplitude and time period of oscillations versus the CME attributes. We do not present diagrams for the space period of oscillation because it depends on the space period. The diagrams do not reveal any characteristic dependencies or correlations. We find only a very poor correlation between the velocity of CMEs and the amplitude of oscillations (panel a-a, correlation coefficient 0.2 ), and also between the velocity of CMEs and time period of oscillations (panel b-a, correlation coefficient is equal -0.3 ). If oscillations are excited during eruption then their amplitudes should depend on the power of the eruption. This may be revealed by this correlation. However, the diagrams demonstrated that the properties of the speed fluctuations are poorly related to the propagation of CMEs in the interplanetary medium. This is not surprising because we expect that oscillations are normal modes determined by the interior properties of flux ropes (Chen, 
(a) $1996-2004$, cor $=0.2$, \# of CMEs $=773$

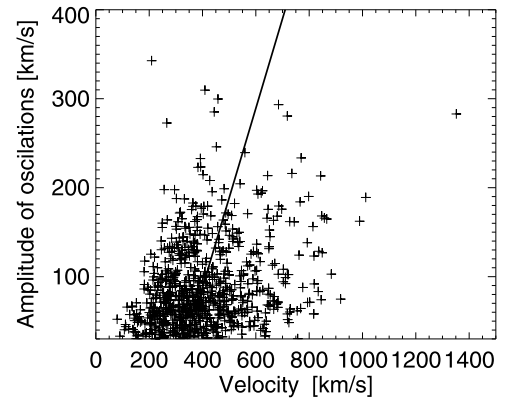

(c) $1996-2004, \mathrm{cor}=0.01$, \# of CMEs $=773$

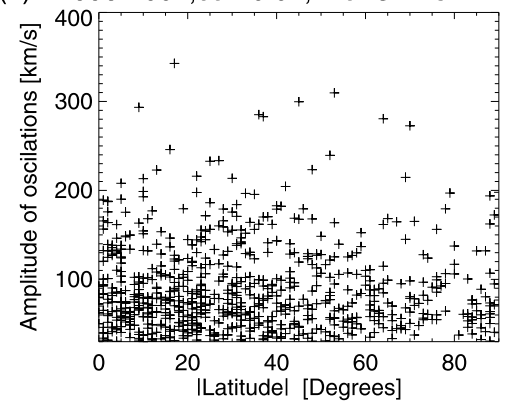

(b) $1996-2004, \mathrm{cor}=0.01$, \# of CMEs $=773$

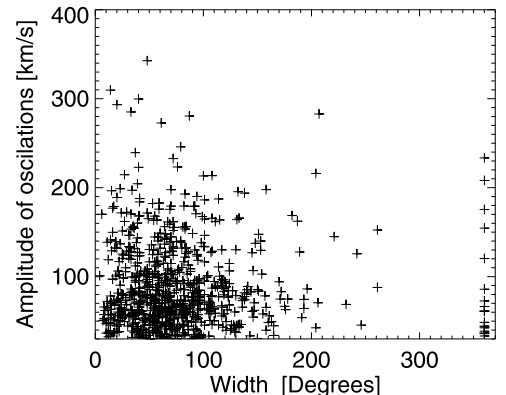

(d) $1996-2004, \mathrm{cor}=0.01$, \# of CMEs $=773$

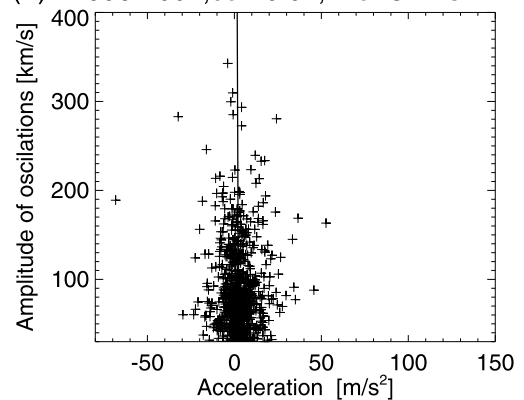

(a) Amplitude versus CME attributes.

(a) $1996-2004, \mathrm{cor}=-0.3$, \# of CMEs $=773$

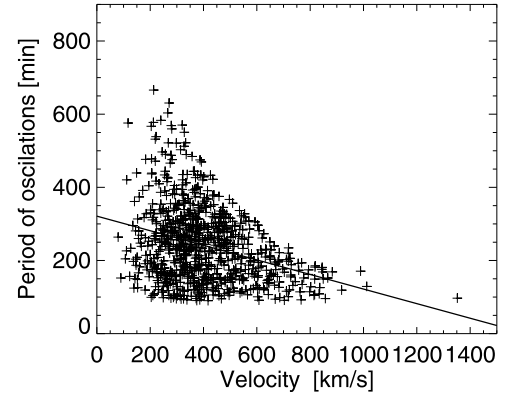

(c) $1996-2004$, cor $=0.05$, \# of CMEs $=773$

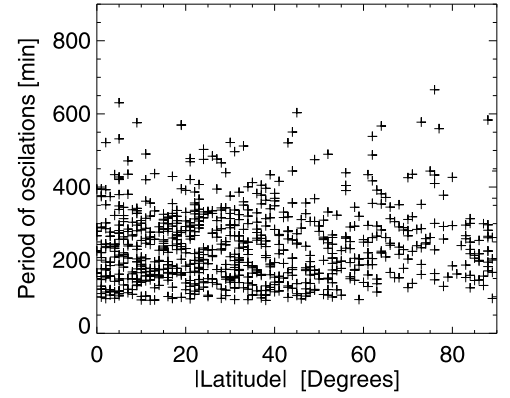

(b) $1996-2004, \mathrm{cor}=0.03$, \# of CMEs $=773$

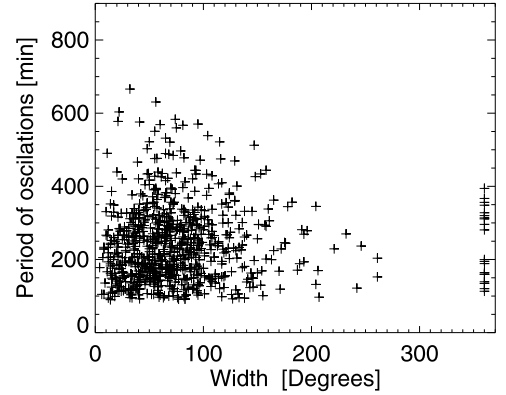

(d) $1996-2004, \mathrm{cor}=0.1$, \# of CMEs $=773$

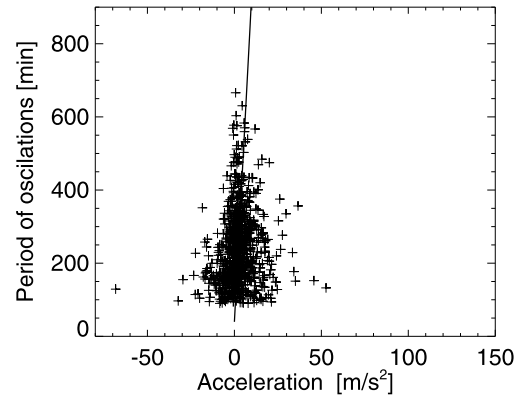

(b) Time period versus CME attributes.

Figure 7 Oscillation attributes versus CME attributes. 

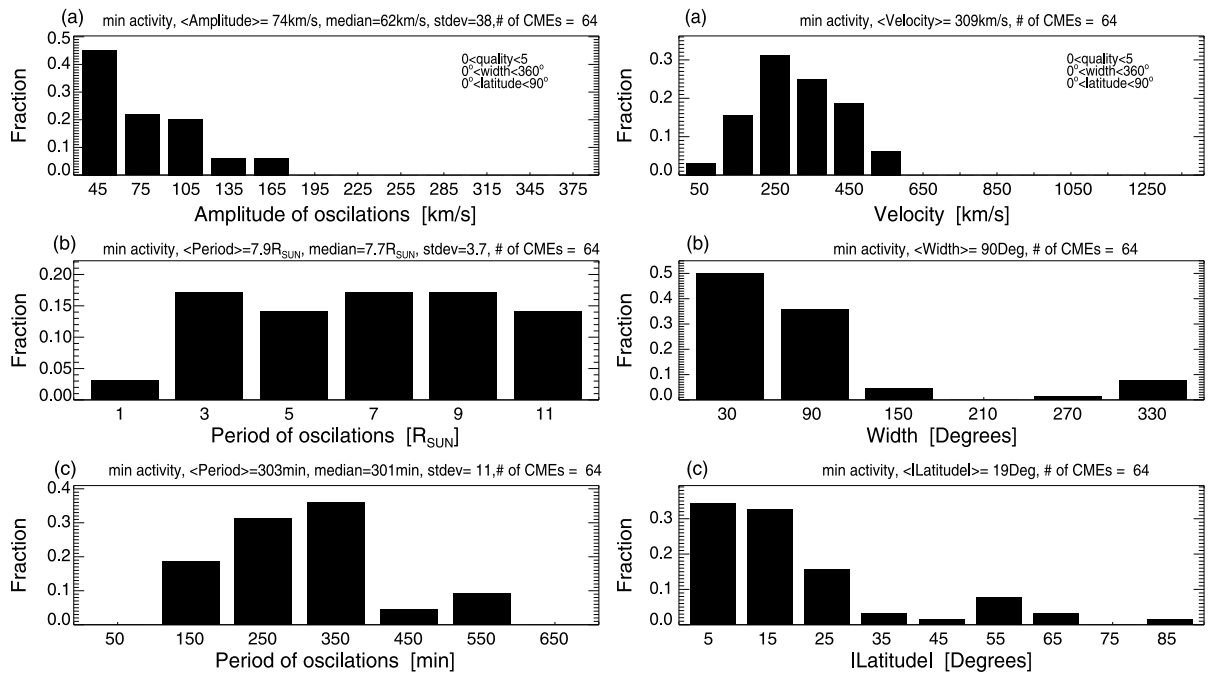

(a) Distributions of oscillation attributes during the minimum of solar activity.

(b) Distribution of CME attributes during the minimum of solar activity.
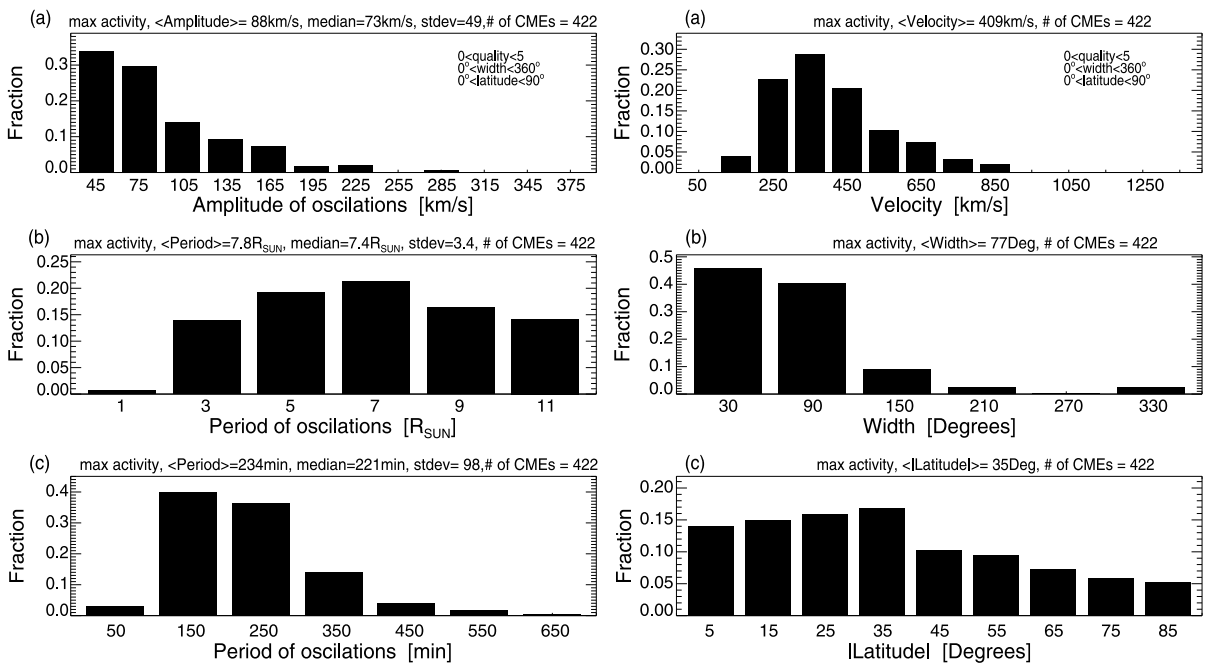

(c) Distributions of oscillation attributes during the maximum of solar activity.

(d) Distributions of CME attributes during the maximum of solar activity.

Figure 8 Distributions of oscillation and CME attributes during the minimum of solar activity (1996 - 1998) and the maximum of solar activity $(2000-2002)$.

1989; Cargill, Chen, and Garren, 1994). This means that the period of oscillation is mainly determined by the strength of the interior magnetic field (see Section 4.1).

\subsection{Comparison Between Minimum and Maximum of the Solar Activity Cycle}

Figure 8 compares the properties of the speed oscillations and associated CMEs in two different periods of the solar activity cycle (minimum of solar activity 1996-1998, maximum 
(a) limb events, $<$ Amplitude $>=93 \mathrm{~km} / \mathrm{s}$, median $=80 \mathrm{~km} / \mathrm{s}$, stdev $=50$, \# of CMEs $=187$

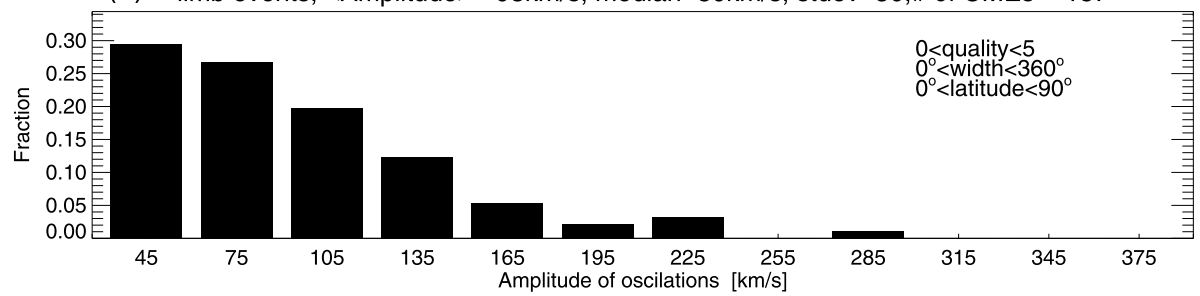

(b) limb events, $<$ Period $>=8.4 \mathrm{R}_{\mathrm{SUN}}$, median $=8.4 \mathrm{R}_{\mathrm{SUN}}$, stdev=3.4, \# of CMEs $=187$

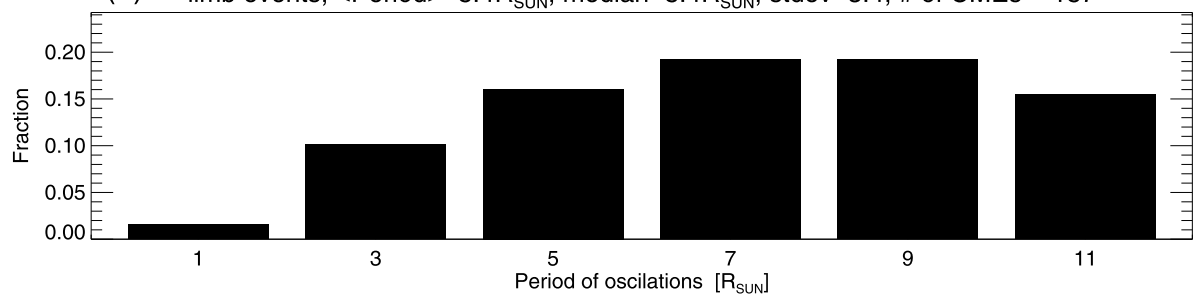

(c) limb events, $<$ Period $>=238 \mathrm{~min}$, median=230min, stdev $=93$, \# of CMEs $=187$

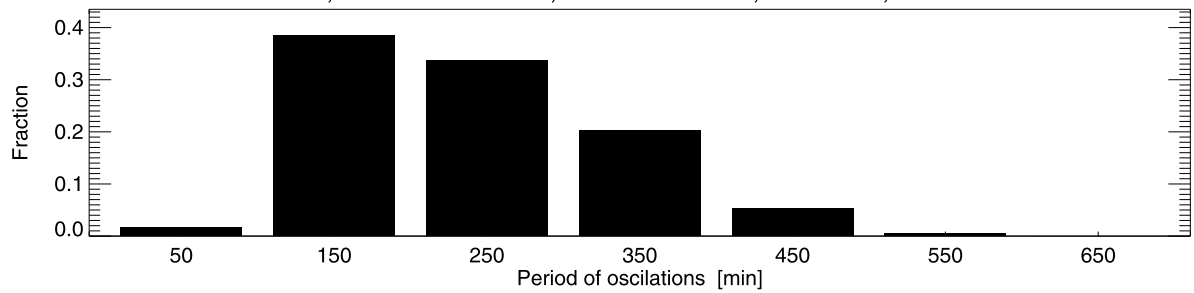

Figure 9 Distributions of the most important attributes of speed oscillations for limb events (|longitude $\mid \geq 60^{\circ}$ ).

of solar activity 2000-2002). The diagrams demonstrate that the parameters of the speed oscillations depend on the solar activity cycle. During the minimum of the solar activity speed oscillations have on average slightly smaller amplitudes (about $14 \mathrm{~km} \mathrm{~s}^{-1}$ smaller) and significantly longer periods of oscillations (by more than an 1 hour) in comparison to the period of the maximum of solar activity. During the minimum of solar activity CMEs are significantly slower and therefore the ejections are less energetic causing a smaller amplitude of the oscillation perturbations. Differences in the durations of the period should be considered based on the magnetic properties of flux ropes. This is discussed in detail in the next section.

\subsection{Limb Events}

Figure 9 shows the attributes of oscillations for limb events. The limb events (having $\mid$ longitude $\mid \geq 60^{\circ}$ ) are free from projection effects and should be more likely to show speed oscillations. In our sample of events we selected 187 limb events. This represents $20 \%$ of all the oscillating CMEs. This fraction is very similar to the fraction of limb events reported in a general population of CMEs. Compared to all the oscillating CMEs, the limb events have, on average, slightly larger amplitudes (by about $6 \mathrm{~km} \mathrm{~s}^{-1}$ ), larger space periods (by about $1 R_{\odot}$ ) and almost the same time periods of oscillations. This is consistent with expectation. The time period of an oscillation is independent of the projection effect and has the same 
value for both sub-samples of CMEs. The two other attributes of oscillation depend on projection effects, and they are slightly larger for the limb events (which are free of projection effects).

\section{Discussion}

\subsection{Nature of Speed Oscillations}

CMEs are closed coronal magnetic structures ejected into the interplanetary medium. They have flux rope magnetic structures similar to solar prominences. Periodic velocity and intensity oscillations of prominences have been known for a few decades (e.g. Bashkirtsev, Kobanov, and Mashnich, 1983; Balthasar et al., 1986). It is assumed that the basic modes of prominence oscillations are similar to the modes of oscillation of a stretched elastic string of non-uniform density (e.g. Leroy, 1989; Kim, 1989). In the case of a prominence the stretched elastic string is a flux rope magnetic structure. Perturbation of a prominence generates string modes being either Alfvén or fast or slow MHD waves (Oliver et al., 1993). Periods of string mode oscillations can be expressed as (Roberts, 1991)

$$
P=\frac{2 \pi}{c_{\mathrm{A}}}(a l)^{1 / 2},
$$

where $a$ is the half-width of the prominence, $l$ is half the length of the flux rope, and $c_{\mathrm{A}}$ is the speed of the MHD waves. This simple formula can explain periodicity in a large number of quiescent prominences. However, in the case of CMEs the situation is more complicated. During their expansion into space, their properties (magnetic field, density and size) change. This means that the normal modes of oscillation also evolve with time. Assuming typical parameters characterizing CMEs at a distance $5 R_{\odot}\left(n=1.6 \times 10^{4} \mathrm{~cm}^{-3}, B=25 \mathrm{mG}\right.$, $l=10 R_{\odot}$ and $a=l / 60$ ), Equation (2) gives the period of Alfvénic oscillation as $220 \mathrm{~min}$, almost the same as the average period of observed oscillations. Dispersion in periods for observed oscillations is due to CMEs having a range of different magnetic properties. Assuming the waves are Alfvén waves we can also explain differences in the period of the observed oscillations during the minimum and maximum phases of the solar activity cycle. During the minimum of solar activity the magnetic fields in active regions are smaller, and therefore the magnetic flux rope structures in CMEs have, on average, a weaker magnetic field inside. If we assume an interior magnetic field of $20 \mathrm{mG}$, then using again Equation (2), we obtain $266 \mathrm{~min}$ for the period of Alfvénic oscillations. This is approximately the same value as the average period for oscillations during the minimum of the solar activity cycle. We have to remember that the period of oscillation depends also on the density and size of the magnetic cloud structure.

\subsection{Conclusions}

We considered a set of 3463 CMEs during the period of 1996-2004 for which 11 heighttime measurements were available. This allowed us to evaluate speed-distance/time profiles with sufficient accuracy to identify and analyze oscillations in the speed of the CMEs. Of the considered CMEs $22 \%$ revealed periodic velocity fluctuations during their expansion in the interplanetary medium. This means that speed oscillation is a common phenomenon associated with CME propagation. However, oscillation attributes depend only poorly on CME 
attributes. This suggests that the properties of speed oscillations must be determined by the magnetic structure of the flux rope. The nature of speed oscillations can be interpreted in terms of normal modes of oscillation of a stretched magnetic string of non-uniform density. CMEs evolve during propagation and so the normal mode also is modified. The average amplitude of speed oscillation is equal to $87 \mathrm{~km} \mathrm{~s}^{-1}$, the average space period and time period are $7.8 R_{\odot}$ and $241 \mathrm{~min}$. The parameters of speed oscillations take a wide range of values (from $0.94 R_{\odot}$ up to $15.97 R_{\odot}$ for space periods, and from 90 up to $824 \mathrm{~min}$ for time periods). The speed oscillations are mostly detected during the maximum of the solar activity cycle when the CMEs are more energetic and can easily excite perturbations which can be detected by LASCO observations. It was demonstrated that the speed oscillations are more likely to be detected for CMEs having a significant quality index. This proves that the speed oscillations are a real physical phenomenon rather than a random numerical effect. More accurate detection of these modes could, in the future, enable us to study the evolution of magnetic structures in space (space seismology).

Acknowledgements Grzegorz Michalek was supported by NCN through the grant UMO-2013/09/B/ST9/ 00034. This work was supported by NASA LWS TR\&T program.

Open Access This article is distributed under the terms of the Creative Commons Attribution 4.0 International License (http://creativecommons.org/licenses/by/4.0/), which permits unrestricted use, distribution, and reproduction in any medium, provided you give appropriate credit to the original author(s) and the source, provide a link to the Creative Commons license, and indicate if changes were made.

\section{References}

Balthasar, H., Knölker, M., Stellmacher, G., Wiehr, E.: 1986, Astron. Astrophys. 163, 343.

Bashkirtsev, V.S., Kobanov, N.I., Mashnich, G.P.: 1983, Solar Phys. 82, 443. DOI.

Cargill, P.J.: 2004, Solar Phys. 221, 135. DOI.

Cargill, P.J., Chen, J., Garren, D.A.: 1994, Astrophys. J. 423, 854. DOI.

Cargill, P.J., Chen, J., Spicer, D.S., Zalesak, S.T.: 1996, J. Geophys. Res. 101, 4855. DOI.

Chen, J.: 1989, Astrophys. J. 344, 1051. DOI.

Chen, J., Krall, J.: 2003, J. Geophys. Res. 108, 1410. DOI.

Chen, J., Howard, R.A., Brueckner, G.E., Santoro, R., Krall, J., et al:: 1997, Astrophys. J. 490, L191. DOI.

Chen, J., Santoro, R.A., Krall, J., Howard, R.A., Duffin, R., et al.: 2000, Astrophys. J. 533, 481. DOI.

Forbes, T.G.: 2000, J. Geophys. Res. 105, 23153. DOI.

Gopalswamy, N., Lara, A., Lepping, R.P., Kaiser, M.L., Berdichevsky, D., et al.: 2000, Geophys. Res. Lett. 27, 145. DOI.

Gopalswamy, N., Lara, A., Yashiro, S., Kaiser, M.L., Howard, R.A.: 2001, J. Geophys. Res. 106, 29207. DOI.

Gopalswamy, N., Yashiro, S., Michalek, G., Kaiser, M.L., Howard, R.A., Reames, D.V., Leske, R., von Rosenvinge, T.: 2002, Astrophys. J. Lett. 572, L103. DOI.

Gopalswamy, N., Yashiro, S., Michalek, G., Stenborg, G., Vourlidas, A., Freeland, S., Howard, R.: 2009, Earth Moon Planets 104, 295. DOI.

Joarder, P.S., Nakariakov, V.M., Roberts, B.: 1997, Solar Phys. 173, 81. DOI.

Kim, I.S.: 1989, In: Ruždjak, V., Tandberg-Hanssen, E. (eds.) Dynamics of Quiescent Prominences, Lecture Notes Phys., Proc. IAU Colloq. 117, Springer, Berlin, 49.

Krall, J.: 2007, Astrophys. J. 657, 559. DOI.

Krall, J., Chen, J., Duffin, R.T., Howard, R.A., Thompson, B.J.: 2001, Astrophys. J. 562, 1045. DOI.

Leroy, J.L.: 1989, In: Priest, E.R. (ed.) Dynamicd and Structure of Quiescent Prominences, Kluwer Academic, Dordrecht, 77.

Lindsay, G.M., Luhmann, J.G., Russell, C.T., Gosling, J.T.: 1999, J. Geophys. Res. 104, 12515. DOI.

Markwardt, C.B.: 2009, Non-linear least squares fitting in IDL with MPFIT. In: Bohlender, D., Dowler, P., Durand, D. (eds.) Proc. Astronomical Data Analysis Software and Systems XVIII, Quebec, Canada, ASP Conf. Ser. 411, Astron. Soc. Pac., San Francisco, 251. ISBN 978-1-58381-702-5.

Oliver, R., Ballester, J.L., Hood, A.W., Priest, E.R.: 1993, Astrophys. J. 409, 809.

Roberts, B.: 1991, Geophys. Astrophys. Fluid Dyn. 62, 83. 
Shanmugaraju, A., Moon, Y.-J., Cho, K.-S., Bong, S.C., Gopalswamy, N., Akiyama, S., Yashiro, S., Umapathy, S., Vršnak, B.: 2010, Astrophys. J. 708, 450. DOI.

Vršnak, B.: 2006, Adv. Space Res. 38, 431. DOI.

Vršnak, B., Ruzdjak, D., Sudar, D., Gopalswamy, N.: 2004, Astron. Astrophys. 423, 717. DOI.

Wang, A.H., Wu, S.T., Gopalswamy, N.: 2005, In: Gallagher, D., Horwitz, J., Perez, J., Preece, R., Quenby, J. (eds.) Particle Acceleration in Astrophysical Plasmas: Geospace and Beyond, AGU Geophys. Monograph 156, 185. DOI.

Wen, Y., Maia, D., Wang, J.: 2007, Astrophys. J. 657, 1117. DOI.

Wood, B.E., Karovska, M., Chen, J., Brueckner, G.E., Cook, J.W., et al.: 1999, Astrophys. J. 512, 484. DOI. Yashiro, S., Gopalswamy, N., Michalek, G., St. Cyr, O.C., Plunkett, S.P., Rich, N.B., Howard, R.A.: 2004, J. Geophys. Res. 109, A07105. DOI. 